\title{
Co-simulation of Power System and Synchrophasor Communication Network on a Single Simulation Platform
}

\author{
Dinesh Rangana Gurusinghe ${ }^{1}$ - Saranga Menike ${ }^{2}$ - A. I. Konara ${ }^{1}$. \\ Athula D. Rajapakse $^{1}$ - Pradeepa Yahampath ${ }^{1}$. U. D. Annakkage ${ }^{1}$. \\ Brian A. Archer ${ }^{3}$. Tony Weekes ${ }^{3}$
}

Received: 7 August 2015 / Accepted: 18 December 2015 / Published online: 1 March 2016

(c) Springer Science+Business Media Singapore 2016

\begin{abstract}
Analysis of the impact of communication network infrastructure performance on critical power system applications that rely on wide area synchrophasor measurements can be realistically achieved only through cosimulation of the power system and the synchrophasor communication network. In this paper, a single simulation platform, an electromagnetic transient (EMT) simulation program - PSCAD/EMTDC, was used to implement
\end{abstract}

Dinesh Rangana Gurusinghe

umgurusi@myumanitoba.ca

Saranga Menike

smudiyansela@hydro.mb.ca

A. I. Konara

anupamaishani@yahoo.com

Athula D. Rajapakse

Athula.Rajapakse@umanitoba.ca

Pradeepa Yahampath

Pradeepa.Yahampath@umanitoba.ca

U. D. Annakkage

Udaya.Annakkage@umanitoba.ca

Brian A. Archer

barcher@hydro.mb.ca

Tony Weekes

maweekes@hydro.mb.ca

1 Department of Electrical and Computer Engineering, University of Manitoba, Winnipeg, MB, R3T 5V6, Canada

2 Distribution Engineering Department, Manitoba Hydro, Winnipeg, MB, Canada

3 System Performance Department, Manitoba Hydro, Winnipeg, MB, Canada the detailed models of the components in a wide area synchrophasor network. Using the developed components models, a simple wide area damping control application was co-simulated to demonstrate the impact of communication latency, packet losses, and bit flip errors on the controller performance. It was shown that with the aid of co-simulations, application specific issues can be diagnosed and appropriate solutions can be investigated.

Keywords Co-simulation · Synchrophasor network modeling - Electromagnetic transient (EMT) simulation . Phasor measurement unit (PMU) · Phasor data concentrator (PDC)

\section{Introduction}

Wide area monitoring, protection and control (WAMPaC) of power systems using synchrophasor measurements is emerging as a useful tool for preventing catastrophic events. WAMPaC systems enable real-time observation of an interconnected power system spread over a large geographical area $[1,2]$. As synchrophasors are time synchronized and updated at a faster rate than traditional supervisory control and data acquisition (SCADA), they encompass necessary information to capture dynamic behavior of a power system [3]. Communication is essential for WAMPaC systems, and for certain applications such as close loop controllers and protection functions, the performance of communication is critical. Although the traditional communication systems used for wide area applications are based on point-to-point communication links [4], a ring topology is often advocated for WAMPaC systems to improve the reliability.

Development of algorithms for wide area protection and control invariably involves simulations. At present there are 
no simulation tools that can simultaneously model both a power system and the synchrophasor communication network, which is part of the WAMPaC system. In the absence of proper tools, most power system studies [5] either assume ideal communication links or use ad-hoc approaches such as using stand-alone communication network simulators for analyzing the communication aspects and subsequently incorporating the extracted results such as average communication latency into the power system simulation. Ad-hoc approaches become intractable in Monte-Carlo simulations, which are essential in analyzing the effects of random events in a communication system.

Modeling and simulation of communication networks have been widely studied in recent years, particularly in relation to local area networks, wide area networks and more broadly the Internet $[6,7]$. Previous work on the performance evaluation of communication networks in wide area power systems, using stand-alone communication network simulation software appears in [4, 5, 8-11]. Typically, the most widely used network simulation software in power system studies is the OPNET [12], an industry standard software package for communication network simulation. Other examples include OMNET++ and NS2 [9]. In [8], OPNET Modeler has been used to simulate a large phasor measurement unit (PMU) network in Nordic countries. The goal of that study has been to determine the requirements of transmission system operators, as well as the network delays and packet losses in a network consisting of a large number of PMUs. However, the power system and the communication network are not simulated as a single system. Authors in [9] present performance aspects of the internet protocol (IP) network infrastructures when utilized by continuous PMU data streams and critical intelligent electronic device (IED)/remote terminal unit (RTU) data. They have used OMNET++, an open source communication simulation environment [13] to simulate the communication network and have examined the end-to-end (ETE) latency between PMUs and the phasor data concentrator (PDC) in a synchrophasor network. The results of simulations performed using OPNET Modeler in order to determine the characteristics of communication delays incurred in wide area monitoring and control systems are presented in [10]. A study of data-traffic modeling in power system control applications is presented in [11].

For wide area monitoring applications, communication system performance is normally not critical. However, as wide area control and protection schemes are being developed, the performance of the communication systems becomes critically important. Communication latency, packet losses and bit flip errors affect the power system behavior when there are closed loop systems such as emergency controls and protection schemes based on wide area measurements. The extensive integration of power system and wide area communication infrastructure mandates the importance of studying the interactions between the two types of systems. The electric power and communication synchronizing simulator (EPOCHS) approach [14] and discrete event system simulator (DEVS) approach [15] are two techniques proposed for co-simulation of a power system and the associated communication network. In these approaches, two separate software platforms and techniques have been used to simulate the power system and the communication network, and the results of the two simulations have been subsequently combined using a third party software. In both [14] and [15], they have used NS2 as the communication network simulation software. In [14], authors have used PSCAD/EMTDC as the power system software and used the EPOCHS approach to build the relationship between the power system simulation software and communication network simulation tool. Further in [15], authors have used a power generation and transmission model of a 17 bus system that is based on the IEEE 14 bus system and have solved the power flow and used the DEVS approach to connect with NS2 network simulation software. While the approaches reported in [14] and [15] is a much better than ad-hoc approaches used in earlier studies, implementation requires specific expertise in all software tools involved. Furthermore, the integration of two stand-alone simulations can lead to errors in synchronization of the results.

The objective of this paper is to co-simulate a power system and a synchrophasor communication network in an integrated manner, so as to identify and characterize the impact of the communication network on the operation of wide area protection and control applications. In contrast to previous work in this paper, the power system and the synchrophasor communication network are co-simulated on a single simulation platform, PSCAD/EMTDC, which is an extensively used EMT simulation program in power industry.

\section{Wide Area Synchrophasor Network Architecture}

A typical architecture of a synchrophasor network is shown in Fig. 1. Synchrophasor measurements collected from PMUs at various locations of an interconnected power system are communicated to a PDC located in a control center (CC) over a wide area communication network [8]. The global position system (GPS) is used to synchronize the phasor measurements to a common time base. Performance and reliability of all core components in the synchrophasor network, including the communication network, are critical in application of real-time wide area control, regardless whether system is a discontinuous control schemes against fast phenomena such as wide area emergency control against transient stability or a continuous feedback control such as a wide area damping controller [16]. 


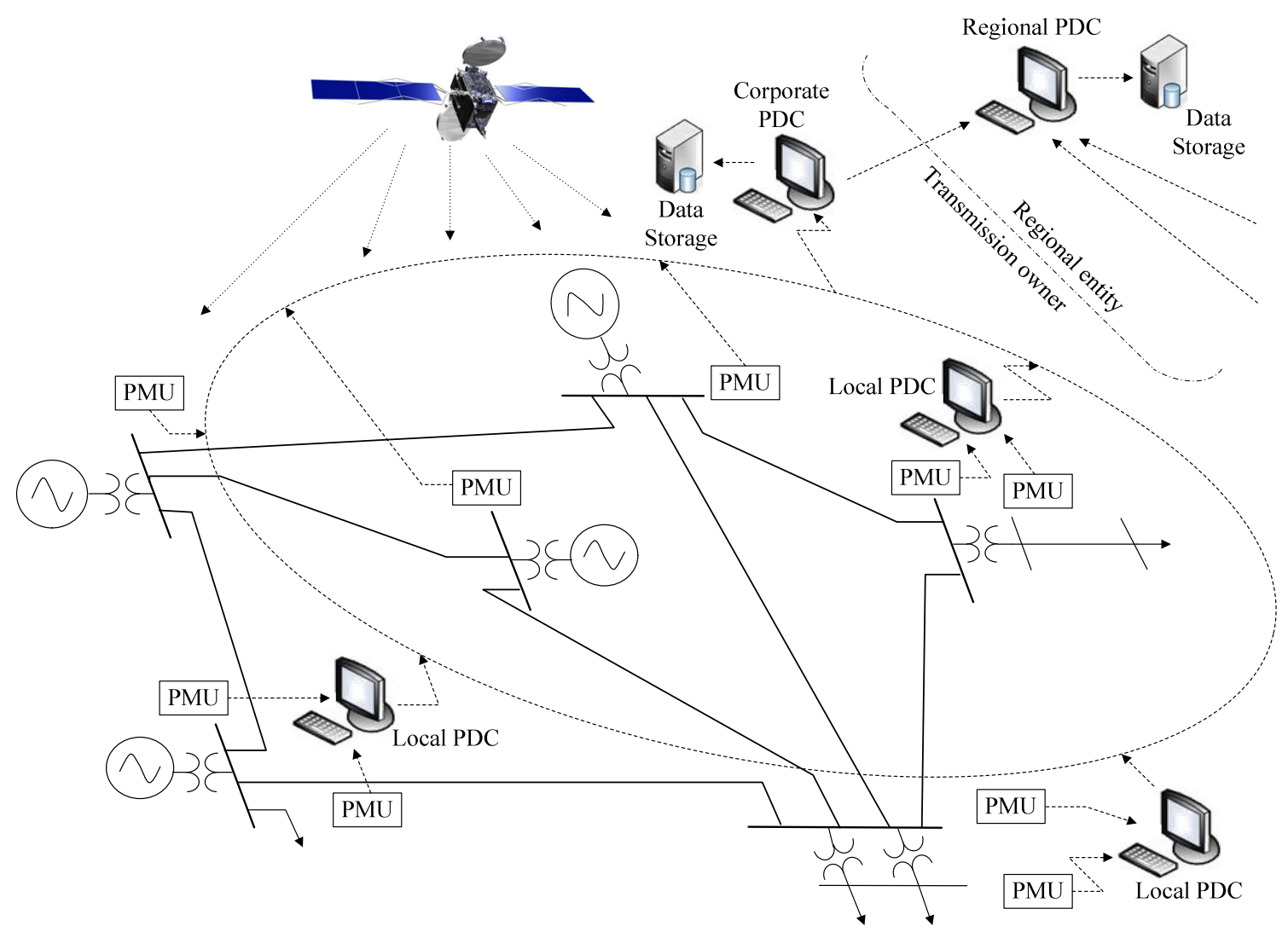

Fig. 1 Typical archtecture of a wide area synchrophasor network

\section{Phasor Measurement Unit (PMU)}

PMUs measure the state variables of important nodes in a power system, and the state variables of other correlated nodes can be calculated with such PMU measurements which enable the observability of the power system [17]. In synchronized phasor measurement, the sampled voltage and current waveforms are projected onto a reference cosine signal synchronized with universal coordinated time (UTC) to extract magnitudes and phase angles of the measured voltage and current signals. A general time varying phasor can be represented as [18],

$X(t)=\frac{X_{m}(t)}{\sqrt{2}} e^{j(2 \pi \Delta f(t) t+\varphi)}=\frac{X_{m}(t)}{\sqrt{2}} \angle(2 \pi \Delta f(t) t+\varphi)$

where $X_{m}(t)$ is the magnitude at time $t, \phi$ is the phase angle measured with respect to a common time reference in such a manner that $\Delta f(t)$ represents the deviation from the nominal power system frequency, $f_{0}$, at time $t$. The measured phasor data are time stamped, and dispatched to the PMU network at a specified rate.

\section{Synchrophasor Communication Network}

A communication network is essential for proper functioning of wide area power system applications. Loss of communication would leave the application with insufficient information to carry out its operations. The communication network used by the control application may be a dedicated network or a part of the corporate data network of the power utility. A corporate network may carry other user traffic (e.g., web pages, e-mail). Hence, the behavior of the wide area control system can be affected by the other uses, and it is important to study the effect of network traffic characteristics on the power system operation.

The reliability, network traffic characteristics and the latency of the communication network are the aspects critical to wide area power system control and monitoring applications. In a packet-based communication network, reliability is related to the packet loss probability and bit errors. Both latency and packet-loss probability depend on the network congestion. The maximum usable data-rate, which is determined by the bandwidth of the communication link, directly impacts the communication latency. 
The delay in a communication link is made-up of several components:

- Multiplexing and transition delay which occur during the exchange of data between a communication link and the data processing equipment

- Propagation delay which is determined by physical channel characteristics (e.g., optical fiber, co-axial cable, or wireless) and the associated physical distances

- Queuing delay which is due to waiting in queues in network components with buffers (e.g., switches, routers)

As more complex and time critical synchrophasor applications evolve, the study of such systems will require synchrophasor network models which can be integrated into power system simulations.

\section{Phasor Data Concentrator (PDC)}

The most important function of a PDC is to collect the phasor measurements from a set of PMUs and align the phasor data according to their GPS time stamp. Collated data are then sent to local applications, higher level PDCs (super PDCs) and archives [19, 20]. If the PMU communication network is to experience abnormal delays or packet losses, some measurements can be lost. A PDC performs quality checks to detect such missing or corrupted data and flags them. Higher level PDCs keep an archive for offline and historical data analysis. The IEEE standard for synchrophasors $[18,21]$ does not specify a protocol to be used for the communication between PMUs and PDCs and specifications for PDCs.

\section{Wide Area Synchrophasor Network Modeling}

A model of a wide area synchrophasor network including the communication system was implemented in the industry standard EMT simulation program, PSCAD/EMTDC. With the implemented models, PSCAD/EMTDC provides a single platform for co-simulation of a power system and a synchrophasor communication network.

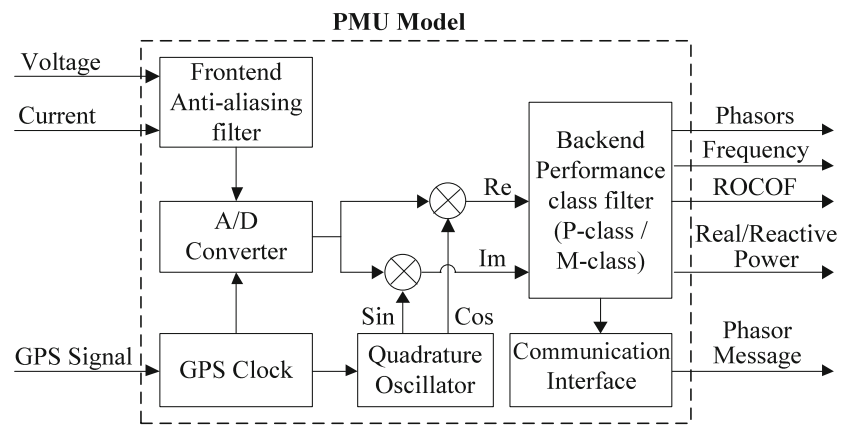

Fig. 2 Model of the PMU

\section{PMU Model}

An accurate model of a PMU that complies with the recently published IEEE standard C37.118.1-2011 [18] was developed in PSCAD/EMTDC software [22]. Main elements in the PMU model are shown in Fig 2. The frontend antialiasing filter (a $6^{\text {th }}$ order Butterworth low-pass filter with a cut-off frequency of half the sampling frequency) eliminates high frequency noise. The signals are digitized at fixed sampling rate, synchronized to a clock signal provide by a GPS clock model. The clock model is initiated with a desired UTC time at the beginning of the simulation and during the simulation, it provides UTC time calculated based on the elapsed time of simulation. The sampling rate of the PMU model can be selected from 8 to 96 samples/cycle. The sampled data points are multiplied by the power system nominal frequency quadrature oscillators to extract real and imaginary components of the phasor, which are then passed through the backend performance class filters (either P-class or M-class) to achieve the required accuracy. Other interested secondary measurements such as frequency, rate of change of frequency (ROCOF) and real/reactive power can be calculated once phasor measurements are available.

The processing delays including group delays of the frontend anti-aliasing filter and the backend performance class filter of the PMU model are corrected with the aid of proper time-stamps, $t_{\text {stamp }}$ which are calculated as,

$t_{\text {stamp }}=t_{U T C}--t_{\text {delay }}$

where $t_{U T C}$ is the current UTC time provided by the clock model and $t_{\text {delay }}$ is the total processing delay of the PMU model. The reporting rate of the PMU model can be selected as per standard reporting rates [18], which are integer submultiples of the system nominal frequency.

In an actual PMU, phasors and other interested quantities including the PMU identification number are combined to make a data frame according to the format prescribed in the standard [21]. This data frame is sent out as a data packet using a communication protocol such as transmission control protocol (TCP)/IP or user datagram protocol (UDP)/IP. In the PMU simulation model, this data packet is represented using an appropriately synthesized set of hexadecimal numbers, since a data frame is too large to represent using a single 64-bit number. The communication interface of the PMU model outputs these numbers and the packet size as parallel messages to be combined and decoded at the PDC model. Performance and accuracy of the PMU model is evaluated under a variety of conditions that are specified in [18] and verification results are provided in [22]. 


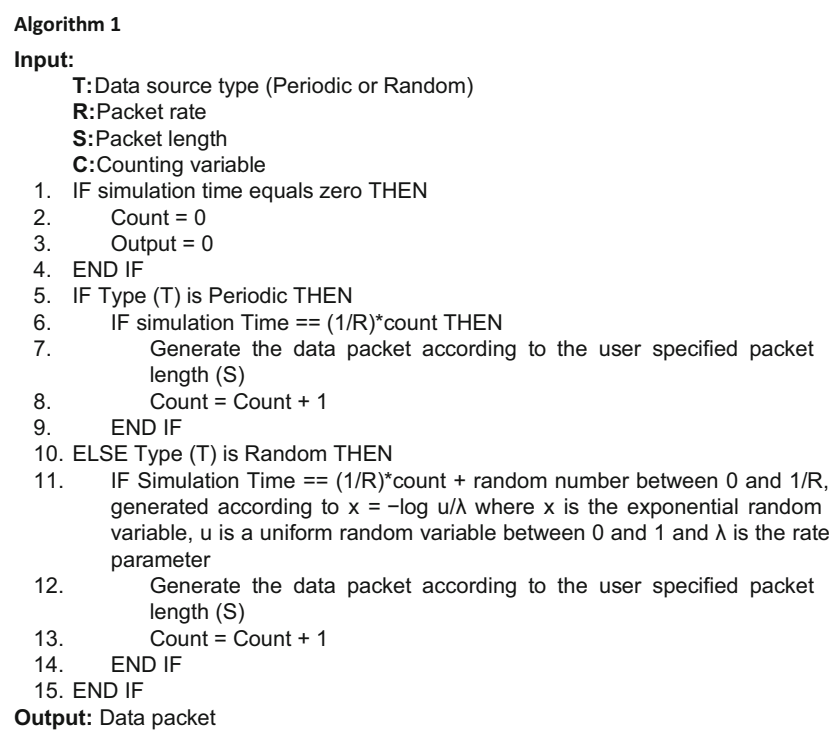

Fig. 3 Data source model

\section{Data Source Models}

Data sources other than PMUs which may share the communication network with PMUs, for example RTUs, video units, protective relays and IEDs were represented using a data source model. When modeling a general data source, the key parameters are the data rate of the source, length of the data packet in bits, and a statistical model for packet generation (periodic, random, etc.). The data source model allows the user to specify the above key parameters. The

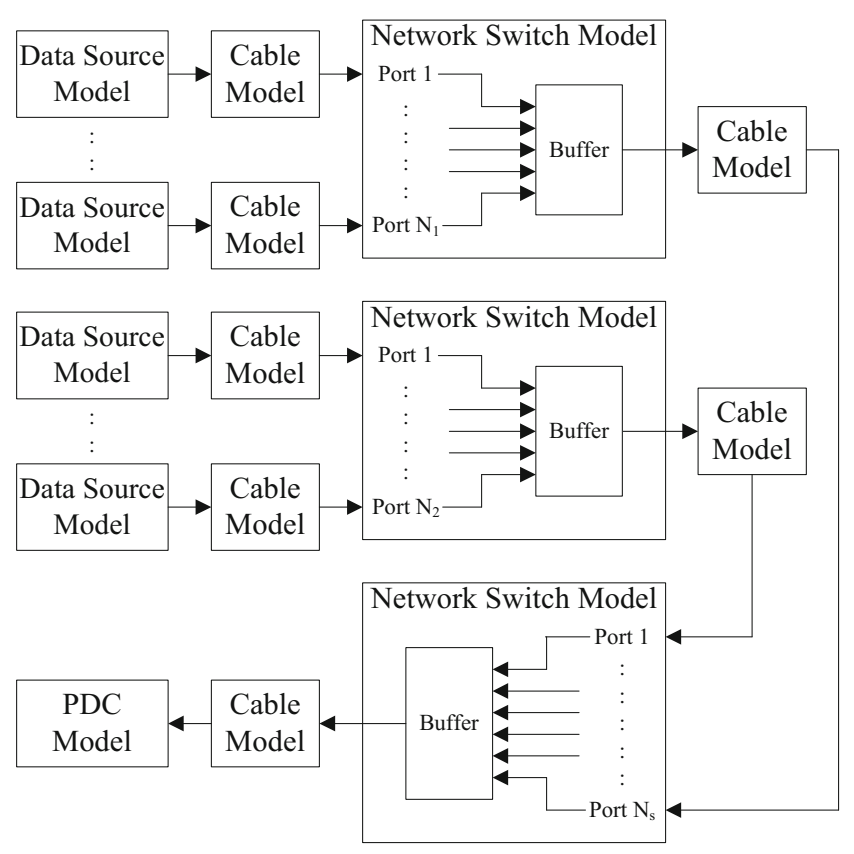

Fig. 4 Schematic of the communication network model

\section{PDC Model}

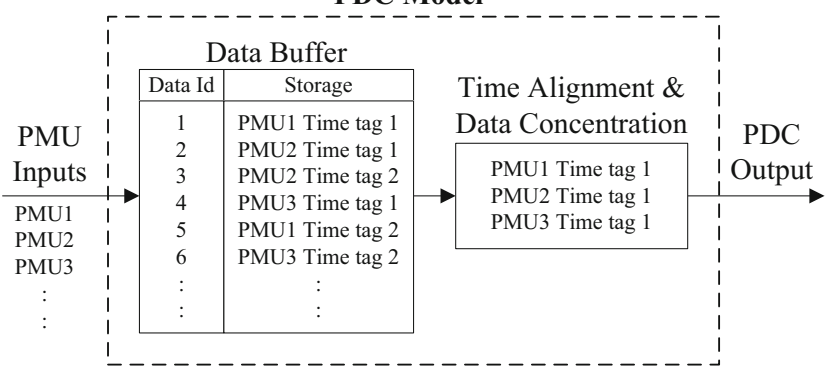

Fig. 5 Model of the PDC

operation inside the data source model is presented in Algorithm 1 given in Fig. 3. The source of information and how they are updated on real time basis in the model is selfexplanatory in the algorithm. Each and every data source is connected to the network switches via cable model.

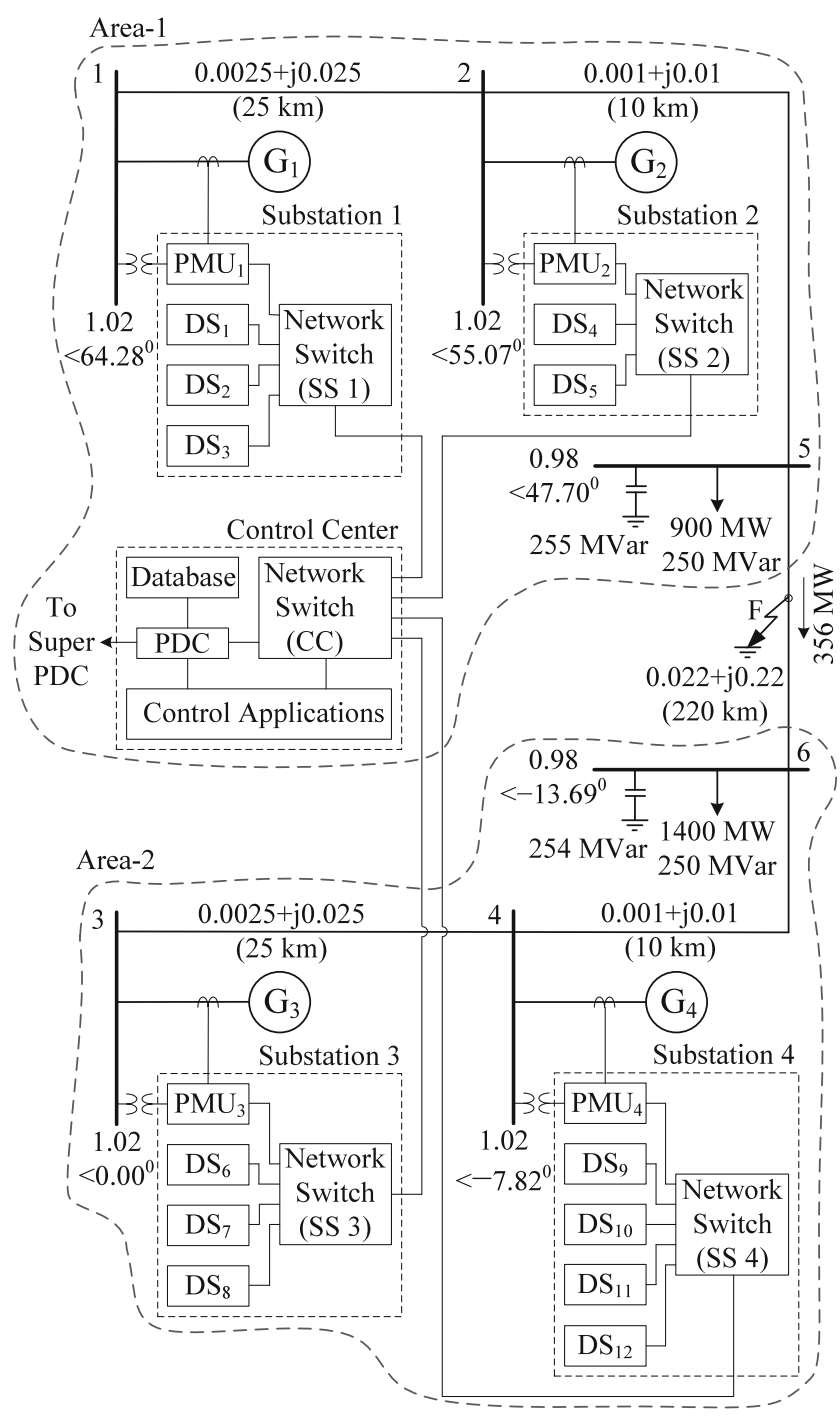

Fig. 6 Simplified two-area four generator power system with the wide area communication network 
Table 1 Initial power flow solution

\begin{tabular}{|c|c|c|c|c|c|c|c|c|}
\hline $\begin{array}{l}\text { Bus } \\
\text { No. }\end{array}$ & $\begin{array}{l}\text { Voltage } \\
(\mathrm{kV})\end{array}$ & $\begin{array}{l}\text { Magnitude } \\
(\mathrm{pu})\end{array}$ & $\begin{array}{l}\text { Angle } \\
(\mathrm{deg})\end{array}$ & $\begin{array}{l}\text { Generation } \\
\text { (MW) }\end{array}$ & (MVar) & $\begin{array}{l}\text { Loads } \\
(\mathrm{MW})\end{array}$ & (MVar) & $\begin{array}{l}\text { Injected } \\
\text { (MVar) }\end{array}$ \\
\hline 1 & 234.6 & 1.020 & 64.28 & 664.0 & -12.9 & -- & -- & -- \\
\hline 2 & 234.6 & 1.020 & 55.07 & 664.0 & 490.9 & -- & -- & -- \\
\hline 3 & 234.6 & 1.020 & 0.00 & 565.1 & -17.7 & -- & -- & -- \\
\hline 4 & 234.6 & 1.020 & -7.82 & 500.0 & 484.1 & -- & -- & -- \\
\hline 5 & 225.1 & 0.979 & 47.70 & -- & -- & 900 & 250 & 244.2 \\
\hline 6 & 224.6 & 0.977 & -13.69 & -- & -- & 1400 & 250 & 242.2 \\
\hline
\end{tabular}

\section{Communication Network Models}

The communication network models comprise of data links, Ethernet communication switches and data sources [23] as shown in Fig. 4. In the model for data link, the user can specify the cable length, the supporting data rate, the desired protocol to be used over the network (either TCP/IP or UDP/IP), the type of cable link (either Ethernet or fiber optic) and the bit error rate of the link. The model simulates the packet delays due to signal propagation over the cable length and the time taken to insert all the bits in an Ethernet frame to the cable. The packet delay, $t_{\text {packet }}$ in a cable link can be defined as,

$t_{\text {packet }}=t_{\text {prop }}+L / R$

where $R$ is the data rate and $t_{\text {prop }}$ is the propagation delay calculated according the user specified cable type and length. The number of bits in the frame, $L$ can be calculated as,

$L=N_{P M U}+N_{T C P / I P}+N_{I P}+N_{\text {transport }}$

where $N_{P M U}, N_{T C P / U D P}, N_{I P}$ and $N_{\text {transport }}$ represent the number of bits in the PMU data message, TCP or UDP header, IP header, and transport layer header and trailer respectively. Furthermore, the model also simulates random bit flip errors according to the user specified bit error rate.

In the model for Ethernet switch, user can specify the buffer size and the processing time of the data packets in the buffer, i.e., switch fabric processing time. The switch model simulates the delay due to the processing time, queuing in the switch buffers, and packet losses due to switch buffer overflow.

The accuracy and the consistency of the communication network models were verified for a simple network by comparing the communication latency and packet loss probabilities obtained with an analytical method based on queuing theory [23].

\section{PDC Model}

The PDC model filters PMU measurements among data stream from PMUs and other data sources. It buffers measurements and waits for pre-assigned time to receive measurements from all PMUs. Thus, the PDC model has a constant processing delay. After the waiting period measurements are aligned and validated. If a measurement is not received within the waiting time due to abnormal delay

Table 2 Details of data sources

\begin{tabular}{lllll}
\hline Data source & Type & $\begin{array}{l}\text { Packet rate } \\
\text { (Packets/s) }\end{array}$ & $\begin{array}{l}\text { Packet size } \\
\text { (Bytes) }\end{array}$ & Data type \\
\hline DS $_{1}$ & RTU & 30 & 600 & Periodic \\
DS $_{2}$ & RTU & 30 & 600 & Periodic \\
DS $_{3}$ & Video unit & 200 & 1100 & Random \\
DS $_{4}$ & RTU & 30 & 600 & Periodic \\
DS $_{5}$ & Video unit & 30 & 600 & Random \\
DS $_{6}$ & RTU & 30 & 600 & Periodic \\
DS $_{7}$ & RTU & 30 & 600 & Periodic \\
DS $_{8}$ & Video unit & 200 & 1100 & Random \\
DS $_{9}$ & RTU & 30 & 600 & Periodic \\
DS $_{10}$ & RTU & 40 & 600 & Periodic \\
DS $_{11}$ & IED & 50 & 800 & Periodic \\
DS $_{12}$ & Video unit & 200 & 1100 & Random \\
\hline
\end{tabular}




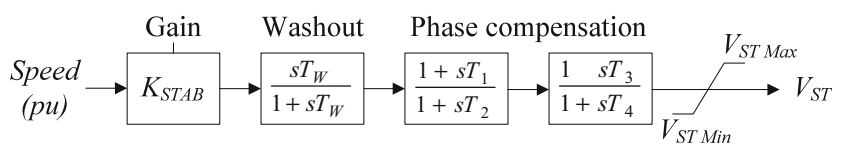

Fig. 7 Speed sensitive power system stabilizer model

or packet loss in the communication network model it is considered as a measurement lost and assigned an error flag. A typical PDC model is shown in Fig. 5.

\section{Results and Discussion}

The importance of co-simulating synchrophasor networks and power systems is demonstrated through a synchrophasor measurement based oscillation damping application on a two-area four generator benchmark power system [24]. A modified version of this system [25] with the wide area communication network is shown in Fig. 6. Each area is comprised of two identical $900 \mathrm{MVA}, 60 \mathrm{~Hz}$, round rotor synchronous machines. All four generators are equipped with IEEE type AC4A exciters [26] and generators are represented using detailed synchronous machine models available in PSCAD/EMTDC master library [27]. Dynamic data for the generator and exciter models were obtained from [25]. The loads are represented as constant admittances and distributed between the areas in such a way that the tie-line transfers $356 \mathrm{MW}$ from Area-1 to Area-2. The simulation is initiated with a power flow solution obtained from PSS/E. The initial power flow solution for all generators and loads is given in Table 1.

All four generator substations are equipped with PMUs that measure the generator terminal voltage and current. The PMU measurements are sent to the local network switch, which also receives information from other data sources that typically appear in substation environment such as RTUs, IEDs and video units [9]. The RTUs and IEDs are considered as periodic packet generation sources while the video units are considered as exponentially distributed random packet generation sources. The type of data source, packet rate, and packet size of each data source are shown in Table 2. The substation network switches are connected to a network switch in the control center, which is assumed to be located near bus-5, a substation with $900 \mathrm{MW}$ load.

The simulated scenario starts with the initial power flow solution and a three-phase bolted fault is applied at the midpoint of the tie-line at $t=1 \mathrm{~s}$ and cleared after 5 cycles. Once the fault is cleared small signal oscillations can be detected from the PMU measurements at the control center. Using a linearized model of the power system around the steady-state operating point, an eigenvalue analysis was conducted to identify the electromechanical modes. The analysis revealed a poorly damped inter-area low frequency oscillatory mode with a frequency of $0.3337 \mathrm{~Hz}$ and a damping ratio of 0.0237 . A power system stabilizer (PSS) was considered for damping these electromechanical oscillations. The PSS function is to modulate the generator excitation system reference to provide a damping torque component in phase with the rotor speed deviation. Therefore, the common input used for PSS is the speed signal (For practical implementation speed signal is derived from the accelerating power). A simple speed sensitive power system stabilizer model [28] is given in Fig. 7. The washout filter prevents the PSS responding to any constant offsets of machine speeds [24]. Lead-lag blocks provide the necessary phase compensation.

The selection of optimal location and inputs for the PSS was carried out based on the calculated residue between exciter reference and the speed of generator. When remote signals are also, considered, the state space system is a multiple input and multiple output (MIMO) system. The residues calculated for this MIMO system in matrix form are

$$
R=\left(\begin{array}{cccc}
2.76 \angle 19.8^{0} & 5.02 \angle 15.4^{0} & 1.56 \angle 153.8^{0} & 4.97 \angle 160.6^{0} \\
2.86 \angle 18.5^{0} & 5.20 \angle 14.1^{0} & 1.61 \angle 155.2^{0} & 5.15 \angle 161.9^{0} \\
6.61 \angle 50.3^{0} & 12.02 \angle 45.9^{0} & 3.72 \angle 123.3^{0} & 11.91 \angle 130.1^{0} \\
6.60 \angle 49.8^{0} & 12.00 \angle 45.4^{0} & 3.72 \angle 123.9^{0} & 11.90 \angle 130.6^{0}
\end{array}\right)
$$

The maximum residue magnitude indicates the best location and the best signal to be used as the input to the PSS. In other words, it gives the maximum sensitivity to feedback gain. It was evident from the calculated residue values that designing a PSS at gen-4 is the best choice for this system under the given operating condition. It was also noted that the use of speed of gen-3 as the feedback signal to the PSS located in gen-4 can be equally effective.

First, a PSS was designed for gen-4 considering its local speed as the feedback signal The parameters $T_{1}$ to $T_{4}$ for the PSS were set to provide phase advance of $47^{\circ}$, so that the eigenvalue pair moves towards the left of the complex plane horizontally. The calculated values are: $T_{1}=T_{3}=0.7251$ $\mathrm{s}$ and $T_{2}=T_{4}=0.3137 \mathrm{~s}$. The gain of the PSS is selected based on the required damping in the system.

The residue between the speed of gen-3 and the exciter reference of gen 4 has a similar residue magnitude and angle. Therefore, by adding this signal to the input of the designed

Table 3 Design parameters for the PSS

\begin{tabular}{lllllll}
\hline Parameter & $K_{S T A B}(\mathrm{pu})$ & $T_{w}(\mathrm{~s})$ & $T_{1}(\mathrm{~s})$ & $T_{2}(\mathrm{~s})$ & $T_{3}(\mathrm{~s})$ & $T_{4}(\mathrm{~s})$ \\
\hline Value & 1.8 & 10.0 & 0.7251 & 0.3137 & 0.7251 & 0.3137 \\
\hline
\end{tabular}


Table 4 Buffer sizes of network switches

\begin{tabular}{llllll}
\hline Network switch & SS-1 & SS-2 & SS-3 & SS-4 & CC \\
\hline Buffer size (pkts) & 100 & 100 & 100 & 1000 & 1000 \\
\hline
\end{tabular}

PSS the sensitivity will be doubled compared to the sensitivity when there is only the speed of gen-4. This could be quite an advantage for a system having a lower residue values as the required controller gains for such a system will be higher. When using multiple inputs to a damping controller the gain required in the controller can be reduced and its reliability increased Therefore, this is a good example case for using a signal measured at a remote location as an input to a PSS. For gen-4, the simple speed sensitive PSS model given in Fig. 7 is used with parameters specified in Table 3 with an input consisting of the sum of the speeds of gen-3 (remote signal) and gen-4 (local signal) The PSS parameters were selected to increase the damping of the system to $15 \%$.

\section{Simulation and Test Results}

A three-phase bolted fault which clears after 5 cycles was applied at the midpoint of the tie-line when time equals to 1.0 seconds. The developed PMU model with M-class filter was used to obtain synchrophasor measurements with a sampling rate of 96 samples/cycle and a reporting rate of 60 frames/s. The data rates of the Ethernet and fiber optic cables were considered as $1 \mathrm{Mbps}$ and $10 \mathrm{Mbps}$ respectively. To avoid packet losses due to the switch buffer over flow, the buffer sizes of each local network switch and the control center switch are set to serve all the packets entering the switch. Buffer sizes of the network switches in the network are given in Table 4. The speed of gen-3, which is measured by $\mathrm{PMU}_{3}$, will be received by the PDC in the control center
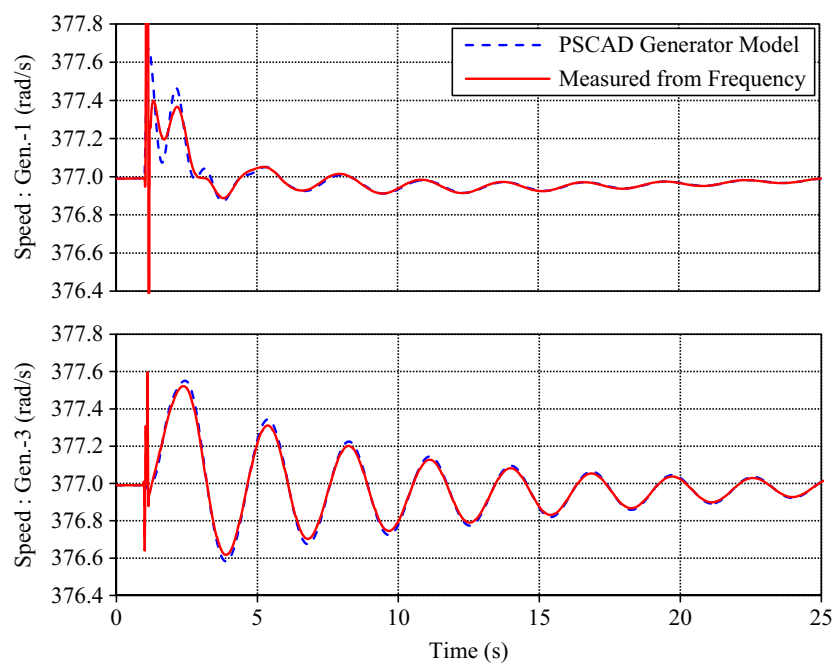

Fig. 8 Speed variations of gen-1 and gen-3 via the communication network. That speed signal will be sent to the PSS installed in gen-4.

\section{Generator Speed Measurement}

Obtaining remote generator speed measurements requires installation of optical or magnetic sensors in the generator and incorporation of the sensor output in the PMU message as an analog data. However, as a more simple and economical alternative, the frequency measurements obtained from the PMU at the remote generator terminal can be used to approximately represent the speed of the generator. The validity of this approach is illustrated in Fig. 8. In Fig. 8, the variations of the speeds of the gen1 and gen-3 obtained directly from the generator model of PSCAD/EMTDC are compared with the generator speeds derived from the frequency measurements of the PMUs. Both the speed obtained from the generator model and the speed derived from the frequency provides the same constant value during the initial steady-state. The speed derived from frequency measurements deviates from the actual speed during the fault, but they converge to the same value within a short period. Therefore, the generator speed derived from the frequency measurements of the PMU can be considered as an acceptable signal to represent the generator speed in a power system damping controller. Here it is assumed that the PMU produces reliable noise-free signal.

\section{Communication Under Normal Operation}

The behavior of the power system under errorless communication (i.e. without bit flip errors and packet losses) is demonstrated in this section. The bit error rates of the cable links were set to be zero, and the power system was

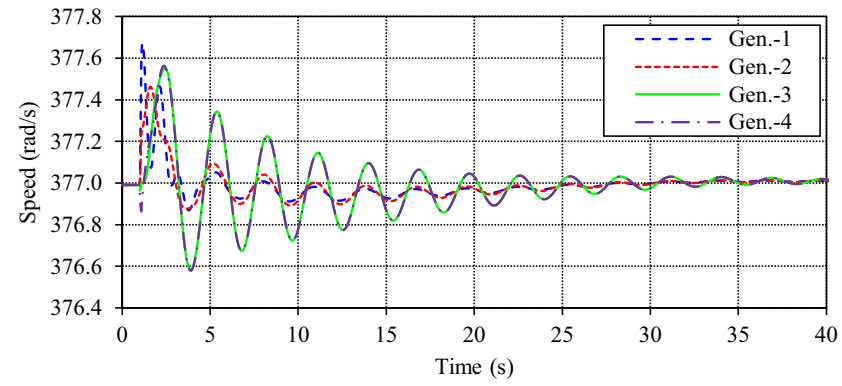

Fig. 9 Speed variation measured from the PSCAD/EMTDC generator model (without a PSS) 
simulated without PSS. Figure 9 clearly reveals the existence of an inter-area oscillation after the three-phase fault on the tie-line. As observed from the measured speed signals, the generators in area-1 are oscillating together with the generators in area- 2 .

The same scenario was simulated twice more, with the PSS at gen-4 enabled. In the first simulation, the PSS was supplied only with a local speed signal input, while in the second simulation, both the local and remote generator speed signals were provided as inputs to the PSS. All speed measurements are derived from the frequency measurement of PMUs and assumed taken from the PDC at the control center. The variations of the speed of gen-3 under three scenarios: (i) without the PSS (to demonstrate small signal oscillations), (ii) with the PSS that uses only the speed of gen- 4 as an input (to demonstrate the damping of oscillations using local signal only), and (iii) with the PSS that uses the speeds of both gen-3 and gen4 as inputs (to demonstrate the damping of oscillations using both local and remote signals) are plotted in Fig. 10. It clearly seen in Fig. 10 that damping is improved after applying the remote signal to the PSS.

Communication latency of the synchrophasor network is illustrated in Fig. 11 for the speed signal of gen-3. It is shown that communication latency is about $1.2 \mathrm{~ms}$, which excludes processing and filtering delays of PMUs, delays of firewalls and the PDC. The authors have observed large latencies such as $300 \mathrm{~ms}$ at a control center mainly due to firewalls, which are not modeled and simulated in this paper. All delays except delays of firewalls are included to the simulations and they do not significantly affect the damping control performance of the PSS. If necessary, the phase shift introduced due to a constant communication latency can be compensated by tuning the PSS [29]. However, communication latency of a synchrophasor network could vary in a random fashion. Therefore, as a compromised solution, the mean latency value can be used to compensate the phase shift of the remote speed signal. As the mean communication latency is about $1.2 \mathrm{~ms}$, which is only a $0.14^{0}$ phase shift for the inter-area oscillation mode

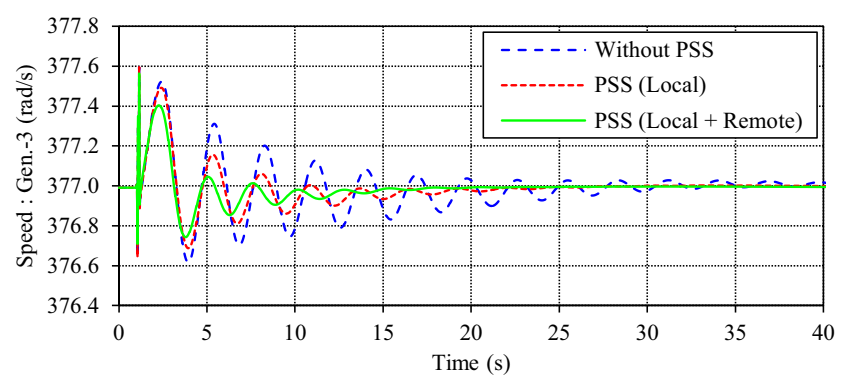

Fig. 10 Speed variation (derived from the PMU model) of gen.-3 measured at the control center ((i) without a PSS, (ii) with a PSS with local speed input and (iii) with a PSS with local and remote speed inputs)

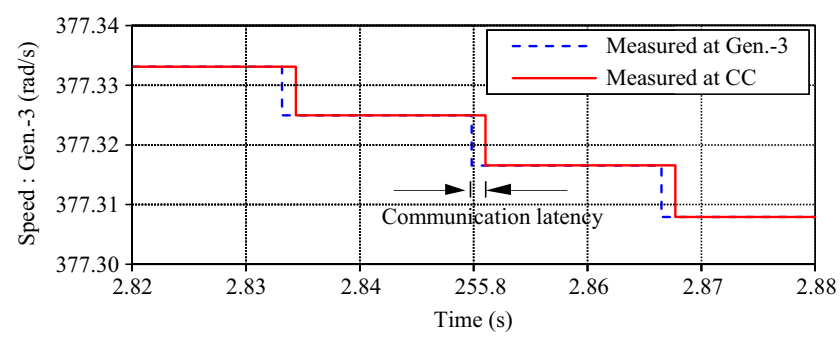

Fig. 11 Speed variation of gen.-3 (remote signal) with communication latency

of $0.3337 \mathrm{~Hz}$ phase compensation is not required in this particular example.

\section{Communication with the Effect of Packet Losses}

In order to simulate data packet losses due to the switch buffer overflow, the packet rate of data source $\mathrm{DS}_{8}$ was increased to very high rate $(99,500 \mathrm{pkts} / \mathrm{s})$ for an interval of one second between $15.0 \mathrm{~s}$ and $16.0 \mathrm{~s}$. This is some unrealistically high jump in the data rate, but it helps in demonstrating the impact of network congestion. Due to congestion in the network switch, some data packets from $\mathrm{PMU}_{3}$ were lost, causing loss of remote input signal to the PSS in random fashion.

This scenario is demonstrated in Fig. 12a, where speed signal shows an abnormal variation due to packet losses causing the power system to become unstable. The level of data storage in buffers and the cumulative packet loss in the network switch, SS-3 is shown in Fig. 12b. It shows that during the interval of $15.0 \mathrm{~s}$ to $16.0 \mathrm{~s}$, switch buffer overflows and data packets are lost. Results indicate that loss of data from the remote generator can have a critical effect on the operation of the PSS, if no remedy is applied:
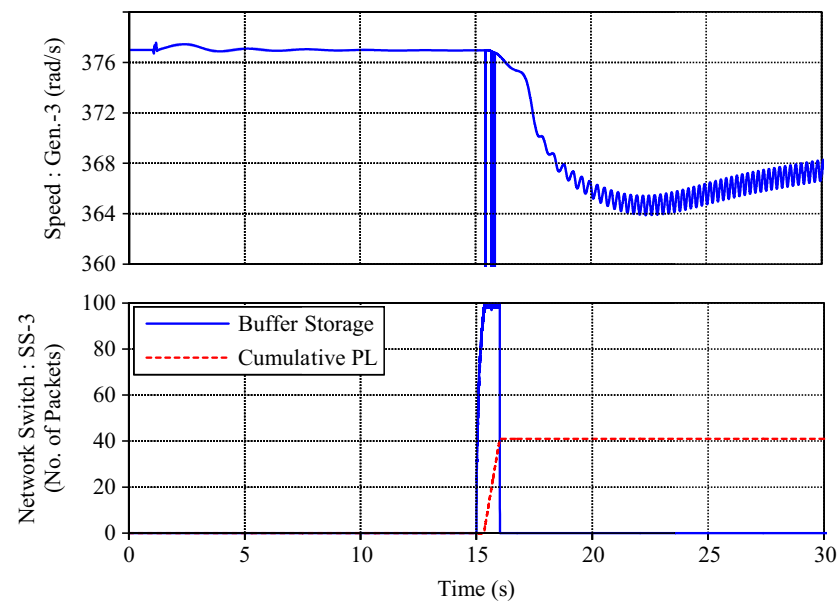

Fig. 12 Effect of single iterval packet losses (a speed variation of gen3 (remote singal) without a control remedy, b SS-3 buffer storage and cumulative packet loss) 
with insufficient input data, PSS can drive the power system into small signal instability. In the above simulation, it is assumed that when new data is not arrived, remote input is set to a default value of zero.

In order to rectify this erratic behavior due to data packet losses several control remedies were investigated. In the first approach, the PSS output limits were narrowed down to \pm 0.05 pu which is a reasonable output limit used on PSSs and it helps to avoid the power system instability. However, this approach still causes unnecessary oscillations in the generator speed and terminal voltage magnitude. In the second approach, the range of variations in the input speed signal was limited to $\pm 5 \%$ of the rated value. This helps to partly reduce the unnecessary oscillations due to data packet losses. In the third approach, the lost data are replaced with the previous measurement. This approach completely eliminated the oscillations triggered by the loss of input data. The variations of the remote speed signal and the terminal voltage of gen-4 under different control remedies are shown in Fig. 13.

In the event of data packet losses, other approaches such as removing the remote signal altogether and reverting back to local input signals could be explored and studied as a possible failsafe mode.

Multi-interval packet losses were applied to further observe the impact of communication network behavior on power system damping control. The data rate of the data source $\mathrm{DS}_{8}$ was increased to 99,500 packets/s for five onesecond intervals starting at $5.0 \mathrm{~s}, 10.0 \mathrm{~s}, 15.0 \mathrm{~s}, 20.0 \mathrm{~s}$ and $25.0 \mathrm{~s}$. Similar to the single interval packet loss case, the power system becomes unstable at the end of first interval as shown in Fig. 14a. The data storage level in buffers and the cumulative packet loss in the network switch, SS-3 are shown in Fig. 14b.
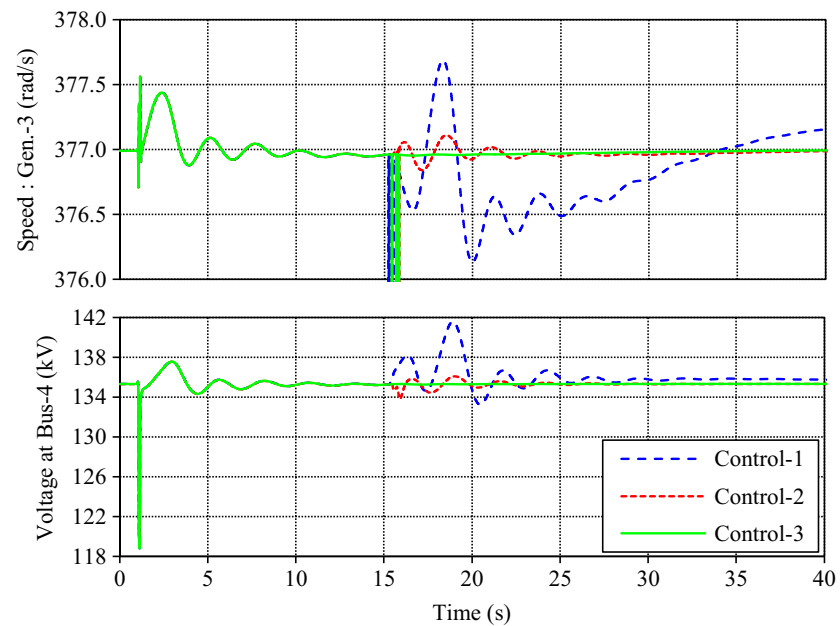

Fig. 13 Effect of single interval packet losses with different control remedies (a speed variation of gen.-3 (remote signal), b voltage variation at bus-4)
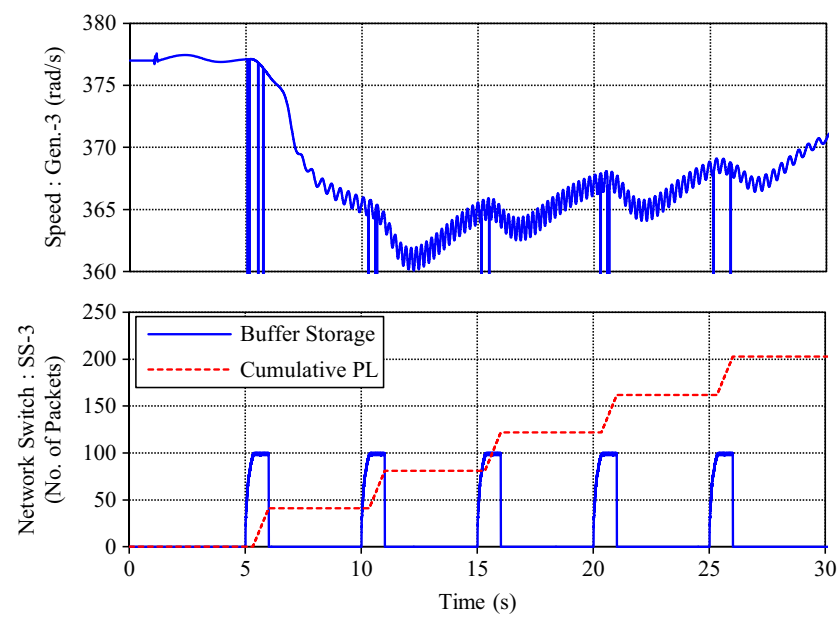

Fig. 14 Effect of multi-interval packet loss (a speed variation of gen.3 without a control remedy, b SS-3 data storage and cumulative packet loss)

The three control remedies discussed earlier were applied again and Fig. 15 illustrates the results in terms of the remote speed signal and the tie-line active power variation. Again, replacement of lost data with the previous measurement is the most appropriate control remedy for this particular application as the oscillation frequency is 0.3337 $\mathrm{Hz}$ and the PMU reporting rate is 60 frames/s.

\section{Communication with the Effect of Bit Flip Errors}

In order to investigate the effect of bit flip errors in the communication links, the bit error rate of the link $(255 \mathrm{~km}$ long fiber optic cable) between network switch SS-3 and network switch CC was set to 0.005 . This is a highly unrealistic value, but helps us to understand the impact of bit errors in
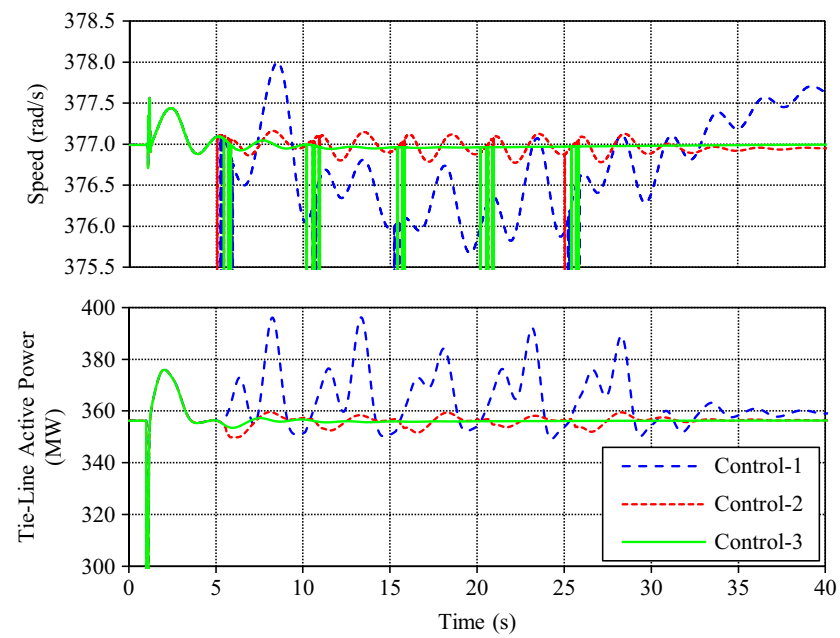

Fig. 15 Effect of multi-interval packet losses with different control remedies (a speed variation of gen.-3 (remote signal), b the tie-line power variation) 


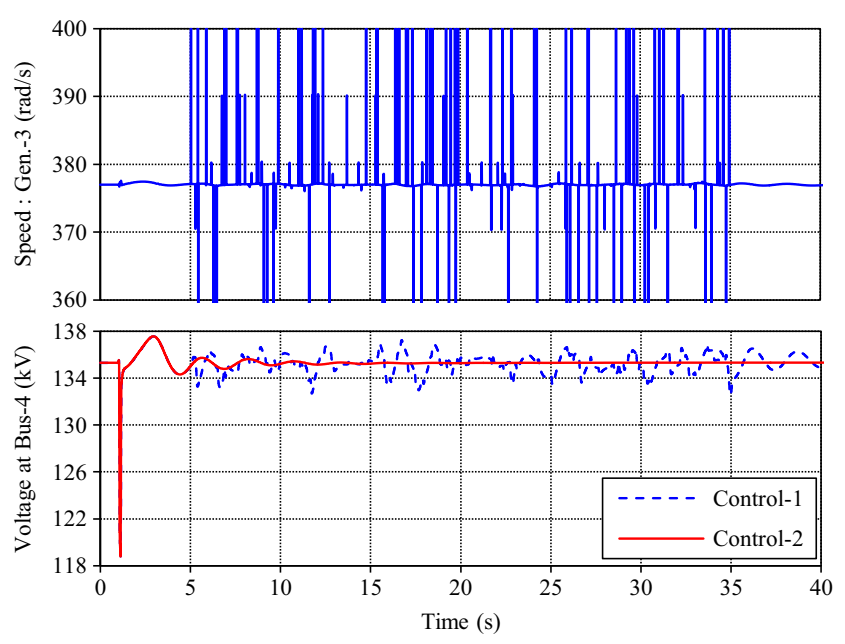

Fig. 16 Effect of bit flip errors with different control remedies (a speed variation of gen.-3 (remote signal), b voltage variation of bus-4)

a short duration simulation. This caused some random bit flips during $5.0 \mathrm{~s}$ to $35.0 \mathrm{~s}$. The results shown in Fig. 16a indicate that bit flip error results can also result in unnecessary oscillations in the power system. Bit errors can occur at any random location in the PMU data frame and any bit flip errors occurring at the bits representing the PMU identification number or the time tag can cause an effect similar to the loss of complete data packet, as the PDC would not be able to recognize the PMU data. Therefore, bit flip errors can be considered as the most critical issue when a synchrophasor signal is used as a remote control signal, as this may not be recognized as an error in some situations.

To eliminate the effect of bit flip errors, the input speed signal range was limited to $\pm 5 \%$ from the nominal speed. The speed of an actual generator is typically within a narrow range under normal operation. Such a remedy can help reduce the unnecessary oscillations but complete elimination of oscillations is impossible. The second control remedy against bit flip errors relies on the fact that the remote control signal, which is the speed of gen-3, cannot be suddenly changed due to large inertia. Therefore, if a large sudden deviation $(> \pm 5 \%)$ in the remote generator speed is detected, the input is replaced with the previous measurement. Figure $16 \mathrm{~b}$ illustrates the variation of terminal voltage of gen-4 (at bus-4) with different control remedies. According to the results, the second approach is a more appropriate control remedy for this particular system, as the generator speed is an inherently slow varying measurement.

\section{Conclusion}

This paper demonstrates that a power system and a synchrophasor communication network can be co-simulated on a single simulation platform. A set of models for simulating synchrophasor network components including the PMUs, network switches, and communication links were developed in the industry standard EMT simulation program, PSCAD/EMTDC. The importance of developed synchrophasor network components were demonstrated through co-simulation of a two area four generator power system, together with a synchrophasor network. The effects of communication latency, bit flip errors and data packet losses for a remote signal based power system oscillation damping control application were demonstrated using the co-simulations. The co-simulations help to diagnose application specific issues related to data communication and determine appropriate remedies. Since the use of fiber optic links were assumed for communication between network switches at the substations and the control center, the effect of the propagation delay of the synchrophasor network was negligible without considering delays of firewall. If it is required and depending on the length of time, the delay can be compensated at the PSS. Modelling of firewalls is beyond the scope of this paper and considered as a future extension. Packet losses and bit flip errors can have a considerable effect on the stability of the power system. It was shown that the effect of data packet losses can be limited by replacing the lost data with the previous measurement, while issues due to bit flip errors can be rectified by substituting the erroneous measurement with the previous value if the error exceeds a specified threshold. In conclusion, co-simulation on a single platform with simulation components developed in this paper is promising and helps to identify the potential bottlenecks of the synchrophasor communication system and optimize the operation of the power system application with the available communication resources.

\section{References}

1. Phadke . AG, Thorp JS, Adamiak MG (1983) A new measurement technique for tracking voltage phasors, local system frequency, and rate of change of frequency. IEEE Trans Power Eng Rev PER-3:23

2. Phadke AG (1993) Synchronized phasor measurements in power systems. IEEE Comput Appl Power:10-15

3. Huang Z, Kasztenny B, Madani V, Martin K, Meliopoulos S, Novosel D, Stenbakken J Performance evaluation of phasor measurement systems. In: Proceedings 2008 IEEE Power Engineering Society General Meeting, Pittsburgh, PA, pp 1-7

4. Chenine M, Karam E, Nordstrom L Modeling and simulation of wide area monitoring and control systems in IP-based networks. In: Proceedings 2009 IEEE Power Engineering Society General Meeting, Calgary, AB, pp 1-8

5. Deng Y, Lin H, Phadke AG, Shukla S, Thorp JS, Mili L Communication network modeling and simulation for wide area measurement applications. In: Proceedings 2012 IEEE Power Engineering Society General Meeting, San Diego, CA, pp 1-6

6. Bertsekas D, Gallager R (1992) Data Networks. In: 2 (ed). Prentice-Hall 
7. Ince N, Brag A (2007) Recent advances in modeling and simulation tools for communication networks and services. Springer Science

8. Chenine M, Nordstrom L (2011) Modeling and simulation of wide-area communication for centralized PMU-based applications. IEEE Trans Power Deliv 26:1372-1380

9. Chenine M, Al Khatib I, Ivanovski J, Maden V, Nordstrom L (2010) PMU traffic shaping in IP-based wide area communication. In: Proceedings $5^{t} h$ Conference on Critical Infrastructure, pp 1-6

10. Chenine M, Nordstrom L (2009) Investigation of communication delays and data incompleteness in multi-PMU wide area monitoring and control systems. In: Proceedings International Conference on Electric Power and Energy Conversion System, pp 1-6

11. Wang Z, Scaglione A, Thomas RJ (2010) Generating statistically correct random topologies for testing smart grid communication and control networks. IEEE Trans Smart Grid:28-39

12. OPNET Inc., OPNET Modeler. [Online]. Available: www.opnet.com

13. OMNET++. [Online]. Available: www.omnetpp.org

14. Hopkinson K, Wang X, Giovanini R, Thorp J, Birman K, Coury D (2006) EPOCHS: a platform for agent-based electric power and communication simulation built from commercial off-the-shelf components. IEEE Trans Power Syst 21:548-558

15. Nutaro J, Kuruganti PT, Miller L, Mullen S, Shankar M Integrated hybrid-simulation of electric power and communications systems. In: Proceedings 2007 IEEE Power Eng. Soc. General Meeting, Tampa, FL, pp 1-8

16. Kundur P, Paserba J, Ajjarapu V, Andersson G, Bose A, Canizares C, Hatziargyriou N, Hill D, Stankovic A, Taylor C, Cutsem T, Vittal V (2004) Definition and classification of power system stability. IEEE Trans Power Syst 19:1387-1401

17. Zhao H (2006) A new state estimation model of utilizing PMU measurements. In: Proceedings International Conference on Power System Technology, pp 1-5

18. IEEE standard for synchrophasor measurements for power systems, IEEE Standard C37.118.1-2011, 2011

19. Martin K, Faris T, Hauer J Standardized testing of phasor measurement units. In: Proceedings 2006 Fault and Disturbance Analysis Conference. Georgia Technology, Atlanta, GA, pp 1-21

20. Gurusinghe DR, Rajapakse AD, Narendra K Evaluation of steadystate and dynamic performance of a synchronized phasor measurement unit. In: Proceedings 2012 IEEE Electrical Power and Energy Conference, London, Ontario, pp 57-62

21. IEEE standard for synchrophasor data transfer for power systems, IEEE Standard C37.118.2-2011, 2011

22. Gurusinghe DR, Rajapakse AD, Muthumuni D Modeling of a synchrophasor measurement unit in an electromagnetic transient simulation program. In: Proceedings 2013 International Conference on Power System Transients, Vancouver, BC, pp 1-8

23. Menike S, Yahampath $P$, Rajapakse AD Implementation of communication network components for transient simulations in PSCAD/EMTDC. In: Proceeding 2013 International Conference on Power System Transients, Vancouver, BC, pp 1-8

24. Kundur P (1994) Power System Stability and Control. McGrawHill, New York

25. Kshatriya N, Annakkage UD, Gole AM, Fernando IT A comparison of the use of participation factors and residues for design and location of power system stabilizers, X SEPOPE, 2006

26. IEEE Committee Report, Excitation system models for power system stability studies, IEEE Transactions on Power Apparatus and Systems, vol. PAS-100, No. 2, pp. 494-509, 1981

27. PSCAD User's Guide, [Online]. Available: https://hvdc.ca/ uploads/ck/files/referencematerial/PSCADUserGuidev431.pdf

28. IEEE recommended practice for excitation system models for power system stability studies, IEEE Standard 421.5-2005, Apr. 2006

29. Chow JH, Sanchez-Gasca JJ, Ren H, Wang S (2000) Power system damping controller design-using multiple input signals. IEEE Control Syst 20(4):82-90

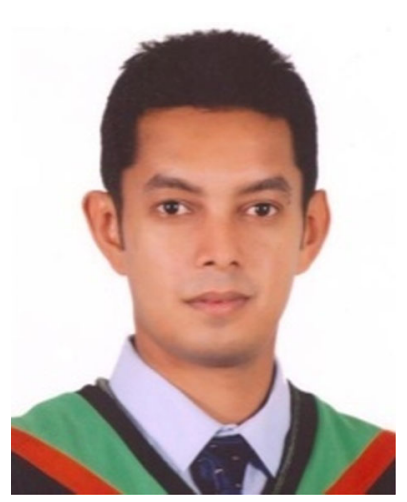

Dinesh Rangana Gurusinghe received the B.Sc. (Eng.) degree in Electrical Engineering from the University of Moratuwa, Katubedda, Sri Lanka, in 2003, the M.Eng. degree in Energy (Electric Power System Management) from the Asian Institute of Technology, Bangkok, Thailand, in 2010, and is currently pursuing the Ph.D. degree in Electrical Engineering with a specialization in Power Systems at the University of Manitoba, Winnipeg, MB, Canada.

He worked as an Electrical Engineer at Cruickshanks Ceylon Ltd, Sri Lanka and Ceylon Electricity Board, Sri Lanka from 2003 to 2008. Since 2015 he is working at RTDS Technologies Inc., Canada as a Simulation Specialist. He is a Corporate Member and a Charted Engineer in the Institution of Engineers, Sri Lanka.

His main research areas are synchrophasor measurements and their applications in power system, real-time digital simulation, power system protection, power system stability, and power system operation and control.

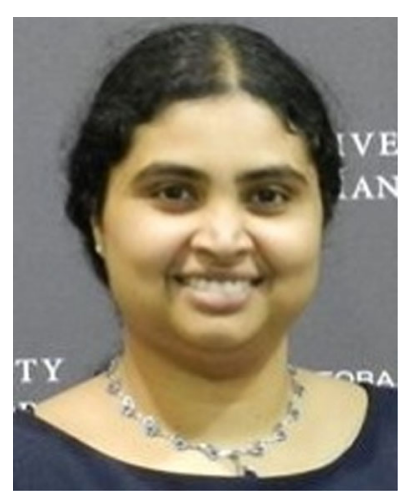

Saranga Menike received the B.Sc. degree from University of Ruhuna, Sri Lanka, in 2009 and the M.Sc. degree from the Department of Electrical and Computer Engineering, University of Manitoba, Canada, in 2013. Currently, she is working as a Planning Engineer in the Distribution Engineering Department in Manitoba Hydro, Canada.

Her research interest includes design and analysis of power system and communication network, power distribution systems, and co-simulation of power System and communication network. Ms. Menike is a Registered Professional Engineer in the province of Manitoba, Canada.

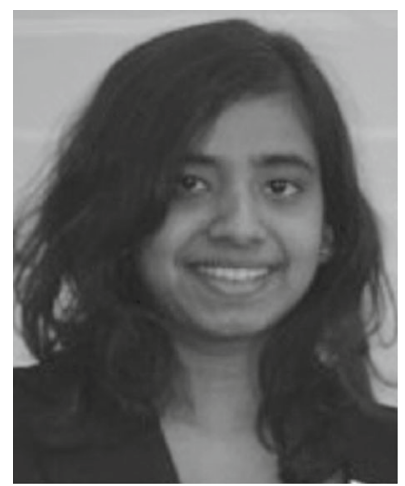

A. I. Konara received the B.Sc. (Eng.) degree from the University of Peradeniya, Peradeniya, Sri Lanka, in 2009 and the Ph.D. degree from the University of Manitoba, Winnipeg, MB, Canada, in 2015.

Her research interests include power system oscillatory stability and design of control algorithms using wide-area measurements. 


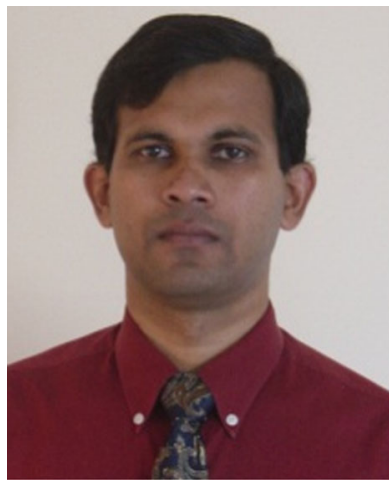

Athula D. Rajapakse received the B.Sc. (Eng.) degree in Electrical Engineering from the University of Moratuwa, Katubedda, Sri Lanka, in 1990, the M.Eng. degree in Energy (Energy Technology) from the Asian Institute of Technology, Bangkok, Thailand, in 1993, and the Ph.D. degree in Quantum Engineering \& Systems Science from the University of Tokyo, Tokyo, Japan, in 1998.

Currently, he is a Professor at the University of Manitoba, Winnipeg, MB, Canada. His research interests include power system protection, grid integration of distributed and renewable energy systems, and protection of future DC grids. Dr. Rajapakse is a Registered Professional Engineer in the province of Manitoba, Canada.

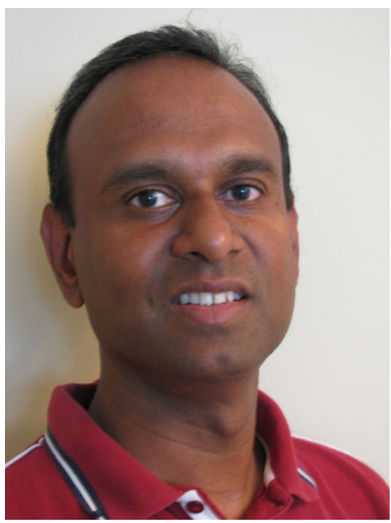

Pradeepa Yahampath received the B.Sc. (Eng.) degree in Electronic and Telecommunications Engineering from the University of Moratuwa, Sri Lanka, in 1990, the M.Sc. degree in Telecommunications from the Norwegian University of Science and Technology, Trondheim, Norway, in 1995, and the $\mathrm{Ph} . \mathrm{D}$. degree in Electrical and Computer Engineering from the University of Manitoba, Winnipeg, Canada, in 2001

Since July 2002, he has been with the Department of Electrical and Computer Engineering, University of Manitoba, where he is currently an Associate Professor. He has held visiting researcher positions at UNIK, University of Oslo, Norway, during 2007-09. His research interests include signal processing in wireless communication and networks.

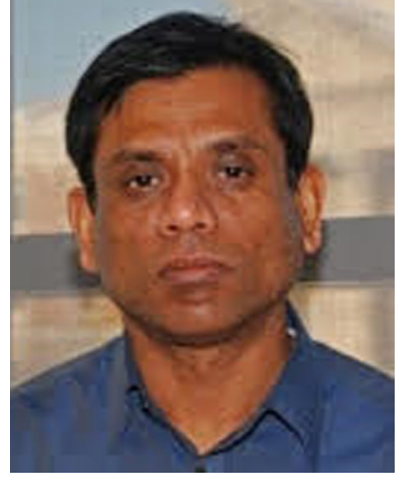

U. D. Annakkage received the B.Sc. (Eng.) degree in electrical engineering from the University of Moratuwa, Moratuwa, Sri Lanka, in 1982, and the M.Sc. and $\mathrm{Ph} . \mathrm{D}$. degrees in electrical engineering from the University of Manchester Institute of Science and Technology (UMIST), Manchester, U.K., in 1984 and 1987, respectively.

$\mathrm{He}$ is presently a Professor with the University of Manitoba, Winnipeg, MB, Canada.

He was the Head of Electrical and Computer Engineering Department, University of Manitoba, from 2008 to 2012. His research interests include power system stability and control, security assessment and control, operation of restructured power systems, and power system simulation.

Dr. Annakkage was an editor for the IEEE Transactions on Power Systems from 2009 to 2012. He is currently the convenor of CIGRE working group on application of phasor measurement units for monitoring power system dynamic performance.

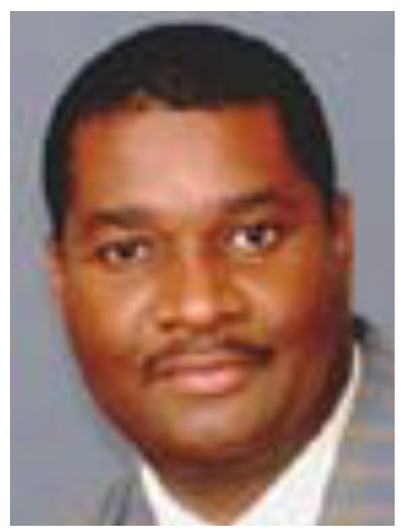

Brian A. Archer obtained his Eng. Tech. diploma and B.Eng. degree in Electrical Engineering from Lakehead University, Canada, in 1988 and 1990 respectively, and his M.Sc. and Ph.D. degrees in Electrical Engineering from the University of Manitoba, Canada, in 1993 and 2008 respectively.

Since 1993 he has worked in various departments within Manitoba Hydro, Canada. He is presently the Protection and Control Performance Engineer (Section Head) in the System Performance Department. He has authored and co-authored several technical papers and participated on many technical panels. His interests include small signal and transient stability, ac and generator controls and HVdc systems and controls. Dr. Archer is a Registered Professional Engineer in the province of Manitoba, Canada, and a member of IEEE. 


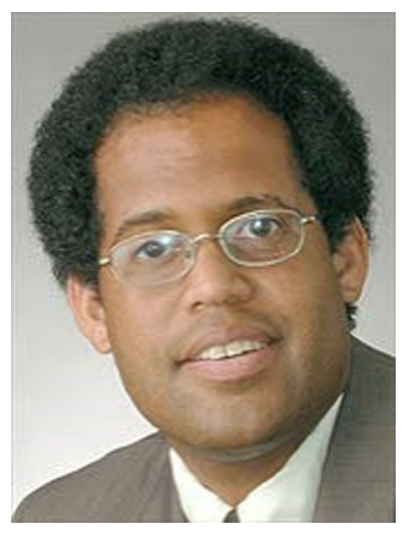

Tony Weekes received his B.Sc. and M.Sc. degrees in electrical engineering from the University of Manitoba, Winnipeg, Manitoba in 1983 and 1986, respectively. His professional career includes positions in HVdc Design, AC System Network Studies, and HVdc System Studies with Manitoba Hydro. Currently, he is the Principal HVdc System Studies Engineer for the System Performance Department of Manitoba Hydro. For the past 10 years Tony has also been the corporate representative for the Eastern Interconnected Phasor Project (now called the North American SynchroPhasor Initiative) which has the goal of providing improved security of the power system through the use of wide area monitoring.

He has over 10 publications and 1 North American patent. His Interests include HVdc controls, Power System analysis, Power system stabilizers, and Wide area measurement systems. He has been a Registered Professional Engineer in the province of Manitoba, Canada for the past 30 years. 